\title{
COMPARACIÓN DE TRES MÉTODOS PARA EL CÁLCULO DE EFICIENCIA DE POZOS
}

\author{
Asdrúbal Vargas ${ }^{1} \&$ Federico Arellano ${ }^{2}$ \\ ${ }^{1}$ Instituto Costarricense de Electricidad \\ ${ }^{2}$ Instituto Costarricense de Acueductos y Alcantarillados \\ E-Mail : avargass@ice.go.cr / farellano@aya.go.cr
}

\begin{abstract}
This work investigated the efficiency of five wells through the development. The efficiency was calculated with the method of Jacob, Rorabaugh and an analitic one. The analytic method was designed by the authors and used to calculate the efficiency with the unknown quantity of the exponent in the expresion $\mathrm{s}=\mathrm{BQ}$ $+\mathrm{CQ}^{\mathrm{n}}$. Three wells were developed using compresed air and two with $\mathrm{CO}_{2}$ (dry ice), generally obtaining an increase in efficiency, but not in all cases. In some cases the efficiency after the development was lower than before, because a well loss was induced through high water discharge. The value of $\mathrm{n}$ is 2 or these about, when the water discharge is low and the flow is laminar. High values of $\mathrm{n}$ were encontered with high water discharge rates.

RESUMEN: En este trabajo se han utilizado los métodos de Jacob, Rorabaugh y analítico para calcular la eficiencia de 5 pozos por medio de 10 pruebas de bombeo a caudal variable, las cuales permitieron medir la efectividad del proceso de desarrollo de los pozos. Se desarrolló un método analítico, que permite calcular la eficiencia de un pozo por medio de ecuaciones donde la incógnita es el valor del exponente en la ecuación $\mathrm{s}=\mathrm{BQ}$ $+\mathrm{CQ}^{\mathrm{n}}$, que forma parte de la componente de pérdida por fricción en el pozo.

De los cinco pozos estudiados tres fueron desarrollados por medio de aire comprimido y 2 con hielo seco, obteniéndose un aumento de la eficiencia prácticamente en todos los casos. El método de Rorabaugh produce valores más elevados de la eficiencia y se debe a que el valor de las pérdidas por fricción en el pozo resultan menores comparadas con el método de Jacob. Se encontró que los valores de n menores o cercanos a 2 se presentan cuando se realizan pruebas de bombeo con caudales relativamente bajos y se tiene un régimen de flujo laminar.
\end{abstract}

\section{INTRODUCCIÓN}

Como parte del diseño geomecánico de un pozo se procede a limpiar y remover las partículas finas a través de un desarrollo de pozos, favoreciendo el reacomodo de los granos del empaque de grava. Una vez desarrollado el pozo, la vida útil del mismo dependerá de varios factores, entre ellos el nivel de corrosión, disminución del área efectiva de entrada en las rejillas o la colmatación del empaque de grava. El desarrollo de los pozos es un procedimiento que consiste en la agitación mecánica del agua por medio de aire comprimido, hielo seco o un pistón dentro del pozo, con el fin de producir corrientes de agua que son impulsadas hacia la formación geológica o el empaque de grava que permiten el acomodo de las partículas y una mejor gradación, así como la limpieza de la rejilla y el aumento de la capacidad de entrada de agua. Este procedimiento puede aumentar la eficiencia de un pozo y con ella la vida útil. 
En este trabajo se cuenta con datos de cinco pozos, ubicados en la región noroeste de Costa Rica (Fig. 1). Tres de ellos han sido desarrollados utilizando aire comprimido (Cuadro 1) y dos con hielo seco. El objetivo de este trabajo es comparar los resultados de los dos métodos de desarrollo de pozos, considerando la eficiencia del pozo antes y después del desarrollo. Además, se pretende estimar la eficiencia del pozo por medio de tres métodos. Por otro lado, se intenta obtener las curvas de rendimiento de los cinco pozos analizados.

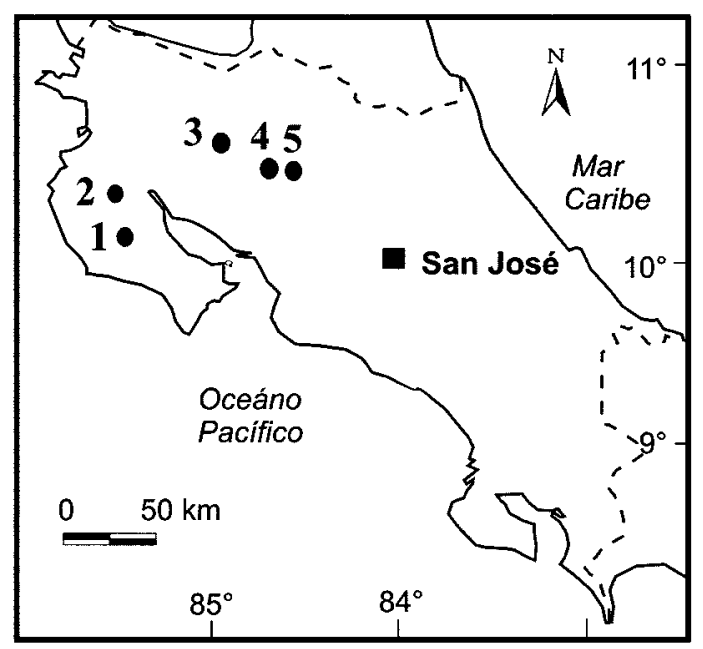

\section{Rio Grande 4 Piedras Verdes 2 Cebadillas 5 Matapalo}

3 Tiquirusas de Porosal

Fig. 1: Ubicación geográfica de los pozos utilizados para realizar las pruebas de bombeo escalonadas.
La metodología de trabajo consistió en la recopilación de información del archivo de pozos del departamento de aguas subterráneas del Instituto Costarricense de Acueductos y Alcantarillados (AyA) y analizar la eficiencia por los métodos de Rorabaugh, Jacob y analítico antes y después del desarrollo de los pozos. El cálculo de la eficiencia antes y después del desarrollo se ha logrado por medio de la aplicación de un programa de computo denominado GWW (Groundwater for Windows), el cual, además, permite definir las curvas de rendimiento del pozo. Los datos de abatimiento y tiempo se graficaron por medio de una hoja electrónica y posteriormente se trasladaron a un programa de dibujo para determinar los abatimientos extrapolados. El modelo analítico de cálculo se desarrolló manualmente y posteriormente se implementó su aplicación por medio de un programa en una calculadora de bolsillo programable.

\section{MÉTODOS DE DESARROLLO DE POZOS}

El desarrollo efectivo de un pozo depende de su profundidad, el tamaño de las rejillas y del tipo de la formación acuífera. Entre los métodos de desarrollo de pozos se encuentran el de agitación, de aire comprimido, hielo seco y agua a presión. El hielo seco o $\mathrm{CO}_{2}$ es una substancia muy utilizada para eliminar las incrustaciones de hierro o de carbonatos en las rejillas y tiene un costo relativamente bajo, pero sólo funciona hasta ciertas profundidades. El desarrollo por medio de aire comprimido requiere de un compresor y la introducción de mangueras dentro de la tubería del pozo.

Cuadro 1

Pozos en funcionamiento desarrollados por medio de aire comprimido (AC) o hielo seco (HS)

\begin{tabular}{lcccccc}
\hline Lugar & Pozo & Coord. & Prof.(m) & Diámetro (mm) & NE (m) & Armado \\
\hline Tiquirusas de Cañas & 1-AC & $408,2-249,30$ & 21,0 & 150 & 5,48 & No hay Inf. \\
Río Grande, Nicoya & 2-AC & $380,3-235,35$ & 27,5 & 150 & 12,76 & 15,5 a 25,5 m rejilla PVC, slot 60 \\
Cebadillas, Santa Cruz & 3-AC & $340,7-248,6$ & 28,0 & 150 & 7,84 & 14 a 27 m rejilla PVC, slot 60 \\
Piedras Verdes, Abangares & 4-HS & $425,8-247,7$ & 16,0 & 150 & 2,00 & No hay Inf. \\
Matapalo, Abangares & 5-HS & $422,6-249,3$ & 43,0 & 200 & 6,05 & No hay Inf. \\
\hline
\end{tabular}

Prof: profundidad del pozo; NE: nivel estático 


\section{Medición de la eficiencia de pozo}

La hidráulica de las captaciones de agua subterránea comprende una serie de conceptos y principios del movimiento del agua subterránea, cuyo objetivo primordial es la predicción del comportamiento del flujo del agua, ya sea en el acuífero o bien en una captación bajo ciertas condiciones dadas. Normalmente se requiere conocer algunas características, tanto del medio como de la captación. Sin embargo, en el caso particular de un pozo profundo, la predicción de los descensos normalmente es inferior que los descensos observados y a medida que se incrementa el caudal de extracción los descensos medidos en el pozo resultan ser aún mayores que los obtenidos teóricamente. Lo anterior se debe a que las fórmulas convencionales consideran solamente un flujo laminar, sin embargo, en las cercanías del pozo generalmente se dan condiciones de flujo turbulento.

Para solventar lo anterior se ha establecido un método para predecir el comportamiento de un determinado pozo, el cual consiste en realizar una prueba a diferentes caudales (ensayo escalonado) y medir sus correspondientes descensos, de tal manera que es posible establecer una fórmula general para un pozo específico. La utilidad de generar esa curva característica del pozo es, que se puede establecer el caudal de explotación óptimo, determinar la eficiencia del pozo y llevar un control de los posibles efectos de colmatación e incrustación en la zona filtrante del pozo (rejilla).

El descenso observado en pozos de bombeo es la suma de los descensos debidos a: a) pérdidas en el acuífero, b) pérdidas por no validez de la ley de Darcy, c) pérdida por entrada al pozo a través de la rejilla, d) pérdidas ascensionales en el pozo y e) pérdidas por entrada en la bomba (Custodio \& Llamas, 1983). El descenso en un pozo puede determinarse según Jacob (1946 en Langguth \& Voigt, 1980) como:

$$
\mathrm{s}_{\mathrm{w}}=\mathrm{BQ}+\mathrm{CQ}^{\mathrm{n}}
$$

donde: $\mathrm{s}_{\mathrm{w}}$ : Abatimiento en el pozo de bombeo $(\mathrm{m})$

Q: Caudal de bombeo $\left(\mathrm{m}^{3} / \mathrm{s}\right)$

B: Resistencia hidráulica del acuífero $\left(\mathrm{s} / \mathrm{m}^{2}\right)$

C: Factor de pérdida de fricción en la estructura del pozo $\left(\mathrm{s}^{2} / \mathrm{m}^{5}\right)$

n: Exponente que puede variar de 1 a 4

El término BQ expresa el descenso por pérdidas en el acuífero y $\mathrm{CQ}^{\mathrm{n}}$ las debidas a los demás factores. B es el coeficiente de pérdidas de circulación en la formación y es variable con el tiempo de bombeo y $\mathrm{C}$ es el coeficiente de pérdidas en el pozo y es independiente del tiempo (Fig. 2). El exponente " $n$ " indica las condiciones de turbulencia o flujo laminar en que se mueve el flujo de agua y toma muchas veces el valor de 2 . Sin embargo, el valor de n no siempre es igual a 2 . Si la velocidad de entrada de agua en el pozo es muy baja puede resultar que $\mathrm{n}$ sea 1 . Para calcular los

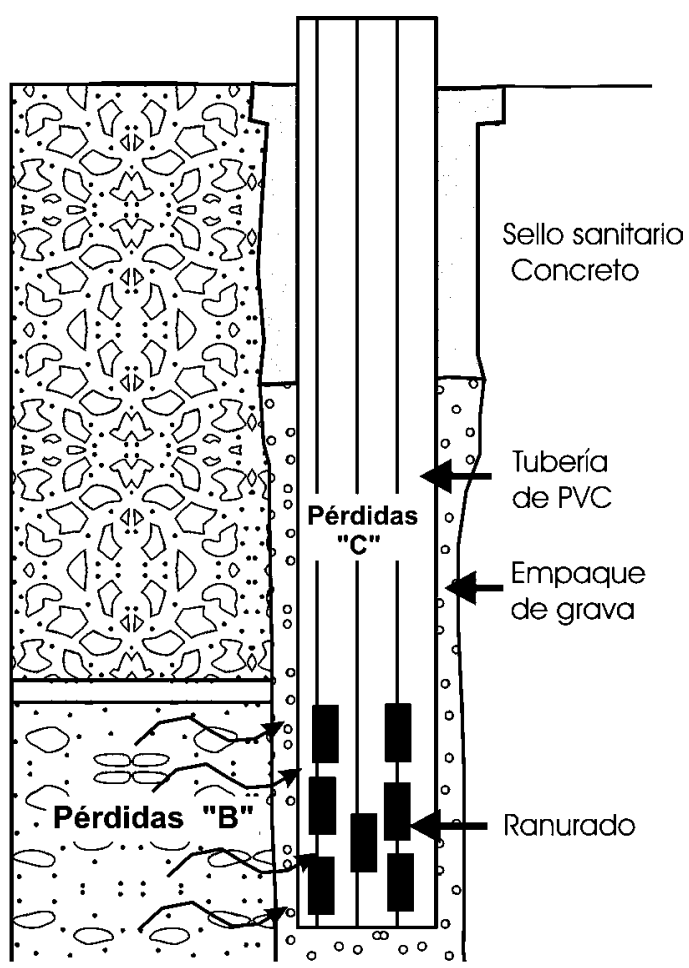

Fig. 2: Esquema de pozo con sus componentes básicos. B representa el factor de pérdidas en la formación y $\mathrm{C}$ el factor por pérdidas en el pozo. 
valores de $\mathrm{B}$ y $\mathrm{C}$, y del exponente $\mathrm{n}$, es necesario conocer los descensos con 2 o 3 caudales diferentes. Los datos de descensos se obtienen de ensayos de bombeo consecutivos a caudal creciente, pero constante en cada intervalo.

Cuando los valores de s/Q vs Q para cada etapa de bombeo se ajustan a una recta, el valor de $\mathrm{n}$ corresponde a 2 y tanto B como C se pueden calcular como la intersección de la recta con el eje Y y el valor de la pendiente de la recta respectivamente. Por otro lado, si se observa que el valor de s/Q es constante, entonces $n=1$ y los descensos son proporcionales a Q lo cual no significa que no existan pérdidas en el pozo. En otro caso se debe calcular $\mathrm{n}$, ya sea por un método de tanteo de Rorabaugh o el método gráfico propuesto por Custodio \& Llamas (1983), o bien a través del método analítico.

La eficiencia se expresa mediante la siguiente expresión:

$$
\text { Eficiencia }=\frac{B \cdot Q}{\left(B \cdot Q+C \cdot Q^{n}\right)} \bullet 100=\frac{s_{\text {teórico }}}{s_{\text {pozo }}}
$$

Si el valor de $\mathrm{C}$ en las etapas finales es considerablemente menor que en las etapas iniciales es probable que ha ocurrido un desarrollo del pozo en las pruebas de caudal variable. Por el contrario, un incremento de $\mathrm{C}$ con un aumento del caudal de bombeo indica la obstrucción de la rejilla (Walton, 1970).

En este trabajo los valores de B y de C, así como de $\mathrm{n}$, se han calculado por medio de un programa de computadora denominado Groundwater for Windows (GWW), desarrollado en 1994 por Dusan Braticevic y Jasminko Karanjac para el Departamento de Desarrollo de la Secretaria de las Naciones Unidas, cuyo propósito es asistir en los estudios hidrogeológicos que se lleven a cabo en centros de investigación. Este programa permite estimar por medio de algoritmos matemáticos los valores de los parámetros mencionados supra utilizando el método de Rorabaugh o de Jacob. Por otro lado, también se muestran los resultados obtenidos con el método analítico.

\section{Pruebas de bombeo por etapas}

La ejecución de pruebas de bombeo previas o posteriores al desarrollo del pozo permite realizar comparaciones en cuanto a la efectividad de dicho proceso en el rendimiento del pozo. El proceso de desarrollo contempla aumentar la porosidad y la conductividad hidráulica de la formación geológica y, además, se busca la remoción y limpieza de sedimentos depositados en la rejilla o que han colmatado el empaque de grava.

\section{MÉTODOS DE ANÁLISIS}

Existen dos métodos clásicos de análisis de la eficiencia, uno de ellos denominado método de Rorabaugh y otro método de Jacob. Además, se desarrolló un método analítico que se puede implementar en una computadora personal o calculadora de bolsillo con resultados muy efectivos.

\section{Método de Rorabaugh}

Es un método de tanteo para encontrar el valor de B. Cada una de las ecuaciones puede transformarse en:

$$
\frac{s}{Q}-B=C \cdot Q^{n-1}
$$

luego:

$$
\log \left(\frac{s}{Q}-B\right)=(n-1) \cdot \log Q+\log C
$$

Si se representa $\log (\mathrm{s} / \mathrm{Q}-\mathrm{B})$ en función de $\log \mathrm{Q}$ se obtiene una recta de pendiente ( $\mathrm{n}$ 1 ), que corta el eje de ordenadas en el valor $\mathrm{C}$. Basta ensayar diferentes valores de B hasta conseguir que los puntos dibujados queden alineados. 


\section{Método de Jacob}

El valor de $\mathrm{n}$ se toma igual a 2, de modo que la ecuación de descensos queda:

$$
\begin{gathered}
s=B \cdot Q+C \cdot Q^{2} \\
s_{1}=B \bullet Q_{1}+C \bullet Q_{1}^{2} ; \quad \frac{s_{1}}{Q_{1}}=B+C \bullet Q_{1} \\
s_{2}=B \bullet Q_{2}+C \cdot Q_{2}^{2} ; \quad \frac{s_{2}}{Q_{2}}=B+C \bullet Q_{2}
\end{gathered}
$$

Se puede efectuar la resolución gráfica, dibujando s/Q en función de Q y trazando la recta que pasa por esos puntos. La pendiente de dicha recta da el valor de $\mathrm{C}$ y la ordenada el valor de $\mathrm{B}$.

\section{Método Analítico}

Se requiere determinar tres variables (B,C,n), por lo tanto se necesitan tres ecuaciones como mínimo, de modo que se hace necesario realizar una prueba con al menos tres caudales diferentes y por supuesto tres descensos diferentes.

$$
\begin{aligned}
& s_{1}=B \cdot Q_{1}+C \cdot Q_{1}^{n} \\
& s_{2}=B \cdot Q_{2}+C \cdot Q_{2}^{n} \\
& s_{3}=B \cdot Q_{3}+C \cdot Q_{3}^{n}
\end{aligned}
$$

Despejar B de (1):

$$
B=\frac{s_{1}-C \cdot Q_{1}^{n}}{Q_{1}}=\frac{s_{1}}{Q_{1}}-C \cdot Q_{1}^{n-1}
$$

Sustituyendo en (2):

$$
\begin{aligned}
& s_{2}=\left(\frac{s_{1}}{Q_{1}}-C \cdot Q_{1}^{n-1}\right) \cdot Q_{2}+C \cdot Q_{2}^{n} \\
& s_{2}=\frac{s_{1} \cdot Q_{2}}{Q_{1}}-\frac{C \cdot Q_{1}^{n} \cdot Q_{2}}{Q_{1}}+C Q_{2}^{n}
\end{aligned}
$$

$$
s_{2}=\frac{s_{1} \cdot Q_{2}}{Q_{1}}+C \cdot Q_{2}\left(Q_{2}^{n-1}-Q_{1}^{n-1}\right)
$$

Despejar B de (2):

$$
B=\frac{s_{2}-C \cdot Q_{2}^{n}}{Q_{2}}=\frac{s_{2}}{Q_{2}}-C \cdot Q_{2}^{n-1}
$$

Sustituyendo en (3):

$$
\begin{gathered}
s_{3}=\left(\frac{s_{2}}{Q_{2}}-C \cdot Q_{2}^{n-1}\right) \cdot Q_{3}+C \cdot Q_{3}^{n} \\
s_{3}=\frac{s_{2} \cdot Q_{3}}{Q_{2}}+C \cdot Q_{3}\left(Q_{3}^{n-1}-Q_{2}^{n-1}\right)
\end{gathered}
$$

Dividiendo (4) y (5):

$$
\frac{s_{2}-\frac{s_{1} \bullet Q_{2}}{Q_{1}}}{s_{3}-\frac{s_{2} \cdot Q_{3}}{Q_{2}}}=\frac{C \cdot Q_{2}\left(Q_{2}^{n-1}-Q_{1}^{n-1}\right)}{C \cdot Q_{3} \cdot\left(Q_{3}^{n-1}-Q_{2}^{n-1}\right)}
$$

$$
\begin{aligned}
& \frac{Q_{1} \cdot Q_{2} \cdot s_{2}-Q_{2} \cdot Q_{2} s_{1}}{Q_{1} \cdot Q_{2} \cdot s_{3}-Q_{1} \cdot Q_{3} \cdot s_{2}}=\frac{Q_{2} \cdot\left(Q_{2}^{n-1}-Q_{1}^{n-1}\right)}{Q_{3} \cdot\left(Q_{3}^{n-1}-Q_{2}^{n-1}\right)} \\
& \frac{Q_{1} \cdot Q_{3} \cdot Q_{2} \cdot s_{2}-Q_{3} \cdot Q_{2} \cdot Q_{2} \cdot s_{1}}{Q_{1} \cdot Q_{2} \cdot Q_{2} \cdot s_{3}-Q_{1} \cdot Q_{2} \cdot Q_{3} \cdot s_{2}}=\frac{\left(Q_{2}^{n-1}-Q_{1}^{n-1}\right)}{\left(Q_{3}^{n-1}-Q_{2}^{n-1}\right)}
\end{aligned}
$$

Dividiendo entre $1 /\left(\mathrm{Q}_{1} \mathrm{Q}_{2} \mathrm{Q}_{2} \mathrm{Q}_{3}\right)$ numerador y denominador:

$$
\frac{\frac{s_{2}}{Q_{2}}-\frac{s_{1}}{Q_{1}}}{\frac{s_{3}}{Q_{3}}-\frac{s_{2}}{Q_{2}}}=\frac{\left(Q_{2}^{n-1}-Q_{1}^{n-1}\right)}{\left(Q_{3}^{n-1}-Q_{2}^{n-1}\right)}
$$

ecuación cuya función solo depende de una incógnita (n).

La ecuación que se presenta puede ser resuelta mediante algún método numérico; en este caso se propone hacerlo con base en la solución numérica de Newton. Este determina la solución de cualquier función $\mathrm{y}=\mathrm{f}(\mathrm{x})$ graficada para $\mathrm{f}(\mathrm{x})$ $=0$ (Fig. 3).

La solución numérica está dada por lo siguiente expresión: 


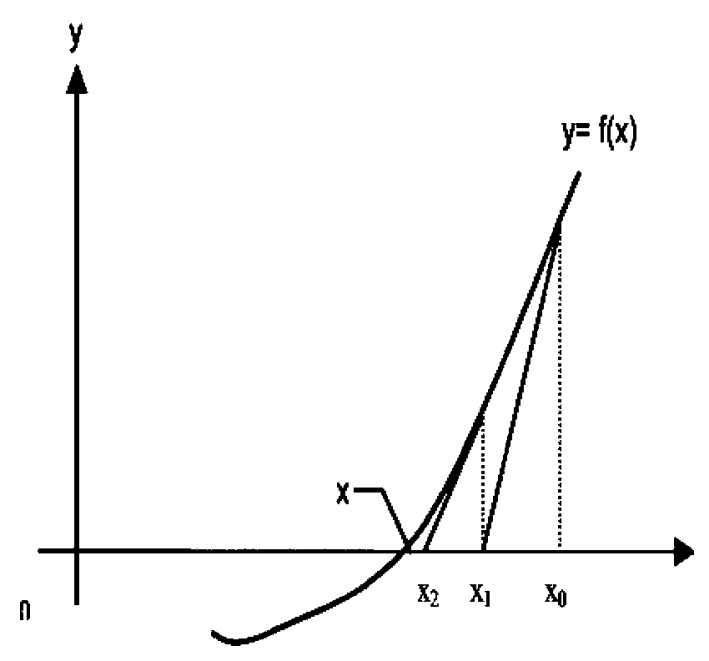

Fig. 3: Función del método analítico de búsqueda del valor del exponente $\mathrm{n}$ en la fórmula $\mathrm{s}=\mathrm{BQ}+\mathrm{CQ}^{\mathrm{n}}$

$$
\mathrm{x}_{0}=\text { Valor inicial }
$$

$\mathrm{h}=$ Intervalo pequeño para el eje $\mathrm{x}$ al efectuar la derivación diferencial numérica en un punto $(\mathrm{x}, \mathrm{f}(\mathrm{x}))$

$$
f^{\prime}(x)=\frac{f(x+h)-f(x)}{h}
$$

$\varepsilon=$ Convergencia de la solución $\left(e>\left|x_{n+1}-x_{n}\right|\right)$ : Calcula el valor de e continuamente y lo retorna mientras la desigualdad sigue siendo verdadera. Para obtener C y B, simplemente despejando de (5):

$$
\begin{gathered}
\left(s_{3}-\frac{s_{2} \bullet Q_{3}}{Q_{2}}\right)=C \cdot Q_{3} \bullet\left(Q_{3}^{n-1}-Q_{2}^{n-1}\right) \\
C=\frac{\frac{\left(s_{3}-s_{2} \bullet Q_{3}\right)}{Q_{2}}}{Q_{3} \bullet\left(Q_{3}^{n-1}-Q_{2}^{n-1}\right)} \\
B=\frac{s_{2}}{Q_{2}}-C \cdot Q_{2}^{n-1}
\end{gathered}
$$

\section{ANÁLISIS DE LAS PRUEBAS DE BOMBEO POR ETAPAS}

Para el análisis de las pruebas de bombeo se cuenta con información de cinco pozos armados con tubería de plástico PVC, rejilla ranurada y bomba sumergible. El empaque de grava consta de arena y grava seleccionada colocada alrededor de la rejilla. La mayoría de los ensayos de bombeo constan de tres etapas de 60 minutos cada una con caudales que varían de $86,4 \mathrm{~m}^{3} / \mathrm{d}$ hasta $259 \mathrm{~m}^{3} / \mathrm{d}$.

\section{Pozo Tiquirusas de Porosal (Cañas)}

Este pozo está ubicado en las coordenadas 408,2/249,3 de la hoja cartográfica Abangares del IGN. La perforación atraviesa una formación sedimentaria, compuesta principalmente por lutitas y areniscas. El tramo productor del acuífero se ubica entre los 6 y 12 metros de profundidad y corresponde a areniscas consolidadas que presentan algún grado de fracturación. No se tiene información detallada del armado del pozo.

Se llevó a cabo un ensayo escalonado de cuatro etapas con períodos de 60 minutos cada etapa, antes y después del desarrollo (Cuadro 2). El cálculo de la eficiencia se ha llevado a cabo con los métodos de Rorabaugh, Jacob y analítico. Por Rorabaugh la eficiencia aumenta después de la limpieza.

Por el método de Jacob hay un descenso de la eficiencia después del desarrollo, debido al abatimiento registrado en el último escalón después del desarrollo en el cual el nivel del agua no alcanzó un equilibrio (Fig. 4). El efecto de este último escalón se observa más claramente si se evalúa la eficiencia después del desarrollo solo con tres etapas, alcanzándose valores del $31 \%$, $23 \%$ y $17 \%$ para cada etapa, respectivamente. Tanto las pérdidas en el acuífero como en el pozo aumentan conforme se aumenta el caudal de bombeo (Cuadro 2). Utilizando el método analítico se observa que la eficiencia aumenta después del desarrollo, a pesar de que los abatimientos diferenciales son mayores. 
Cuadro 2

Parámetros de prueba por etapas en el pozo Tiquirusas de Porosal

\begin{tabular}{|c|c|c|c|c|c|c|c|c|c|}
\hline $\mathrm{Q}\left(\mathrm{m}^{3} / \mathrm{d}\right)$ & Tiempo (min) & sw (m) & swtotal (m) & $\mathrm{n}$ & $\mathrm{B}$ & $\mathrm{C}$ & BQ & $\mathrm{CQ}^{\mathrm{n}}$ & Efic $(\%)$ \\
\hline \multicolumn{7}{|c|}{ Antes del desarrollo 23-5-98 } & \multicolumn{3}{|c|}{ Rorabaugh } \\
\hline 86,4 & 60,0 & 1,1 & 1,1 & 3,22 & 0,0107 & $1,0 \times 10-7$ & 0,92 & 0,18 & 83,8 \\
\hline 172,8 & 60,0 & 2,4 & 3,5 & 3,22 & 0,0107 & $1,0 \times 10-7$ & 1,84 & 1,67 & 52,7 \\
\hline 216,0 & 60,0 & 2,3 & 5,7 & 3,22 & 0,0107 & $1,0 \times 10-7$ & 2,30 & 3,43 & 40,4 \\
\hline \multicolumn{10}{|c|}{ Despúes del desarrollo 25-5-98 } \\
\hline 86,4 & 60,0 & 1,4 & 1,41 & 3,28 & 0,014 & $7,0 \times 10-8$ & 1,21 & 0,16 & 85,7 \\
\hline 172,8 & 60,0 & 2,4 & 3,80 & 3,28 & 0,014 & $7,0 \times 10-8$ & 2,42 & 1,5 & 63,6 \\
\hline 216,0 & 60,0 & 2,4 & 6,21 & 3,28 & 0,014 & $7,0 \times 10-8$ & 3,02 & 3,12 & 48,7 \\
\hline 259,2 & 60,0 & 3,2 & 9,21 & 3,28 & 0,014 & $7,0 \times 10-8$ & 3,83 & 5,67 & 39,4 \\
\hline \multicolumn{7}{|c|}{ Antes del desarrollo 23-5-98 } & \multicolumn{3}{|c|}{ Jacob } \\
\hline 86,4 & 60 & 1,1 & 1,1 & 2,00 & 0,0012 & $1,1 \times 10-4$ & 0,16 & 0,83 & 14,6 \\
\hline 172,8 & 60 & 2,4 & 3,5 & 2,00 & 0,0012 & $1,1 \times 10-4$ & 0,32 & 3,34 & 9,2 \\
\hline 216,0 & 60 & 2,3 & 5,7 & 2,00 & 0,0012 & $1,1 \times 10-4$ & 0,40 & 5,22 & 7,0 \\
\hline \multicolumn{10}{|c|}{ Despúes del desarrollo 25-5-98 } \\
\hline 86,4 & 60 & 1,4 & 1,41 & 2,00 & 0,001 & $1,3 \times 10-4$ & 0,09 & 0,98 & 6,1 \\
\hline 172,8 & 60 & 2,4 & 3,80 & 2,00 & 0,001 & $1,3 \times 10-4$ & 0,17 & 3,91 & 4,5 \\
\hline 216,0 & 60 & 2,4 & 6,21 & 2,00 & 0,001 & $1,3 \times 10-4$ & 0,21 & 6,10 & 3,5 \\
\hline 259,2 & 60 & 3,2 & 9,21 & 2,00 & 0,001 & $1,3 \times 10-4$ & 0,26 & 8,79 & 2,8 \\
\hline \multicolumn{7}{|c|}{ Antes del desarrollo 23-5-98 } & \multicolumn{3}{|c|}{ Mét. analítico } \\
\hline 86,4 & 60,0 & 1,18 & 1,18 & 4,13 & 0,0107 & $6.6 \times 10-10$ & 1,114 & 0,070 & 94,4 \\
\hline 172,8 & 60,0 & 2,20 & 3,38 & 4,13 & 0,0107 & $6.6 \times 10-10$ & 2,228 & 1,148 & 66,0 \\
\hline 216,0 & 60,0 & 2,30 & 5,68 & 4,13 & 0,0107 & $6,6 \times 10-10$ & 2,786 & 2,885 & 49,1 \\
\hline \multicolumn{10}{|c|}{ Despúes del desarrollo 25-5-98 } \\
\hline 86,4 & 60,0 & 1,4 & 1,41 & 4,47 & 0,0160 & $6,5 \times 10-11$ & 1,38 & 0,030 & 97,9 \\
\hline 172,8 & 60,0 & 2,0 & 3,41 & 4,47 & 0,0160 & $6,5 \times 10-11$ & 2,76 & 0,648 & 81,0 \\
\hline 216,0 & 60,0 & 1,8 & 5,21 & 4,47 & 0,0160 & $6,5 \times 10-11$ & 3,45 & 1,758 & 66,2 \\
\hline
\end{tabular}

Q: caudal; sw: abatimiento por etapa; swtotal: abatimiento total acumulado; n: exponente.

Los resultados obtenidos por el método analítico coinciden con los obtenidos por el método de Rorabaugh. Sin embargo, existe una diferencia, producto de que el método de Rorabaugh tomó en cuenta cuatro etapas y el método analítico solamente las tres primeras. El caudal de bombeo adecuado para este pozo es de $172 \mathrm{~m}^{3} / \mathrm{d}$ para obtener un abatimiento de $4 \mathrm{~m}$ (Fig. 5).

\section{Pozo Río Grande de Nicoya}

Este pozo se ubica en las coordenadas 380,3/235,35 de la hoja cartográfica Matambú del IGN. El material que atraviesa la perforación está formado por suelo arcilloso meteorizado proveniente de un relleno coluvio-aluvial entre los $0 \mathrm{y}$ 4 metros de profundidad y por lutitas consolidadas con algún grado de fracturación entre los 4 y 27 metros de profundidad. El pozo presenta rejilla de PVC entre los 15,5 y $25,5 \mathrm{~m}$ de profundidad.

El pozo Río Grande cuenta con dos pruebas de bombeo escalonadas de caudal creciente. La eficiencia del pozo aumenta después del desarrollo según los dos métodos de análisis utilizados, aunque el incremento es mayor por el método de Jacob (Cuadro 3). El abatimiento producido en la última etapa de bombeo es muy pronunciado, pero siempre menor que 1,5 $\mathrm{m}$ en ambas pruebas de 


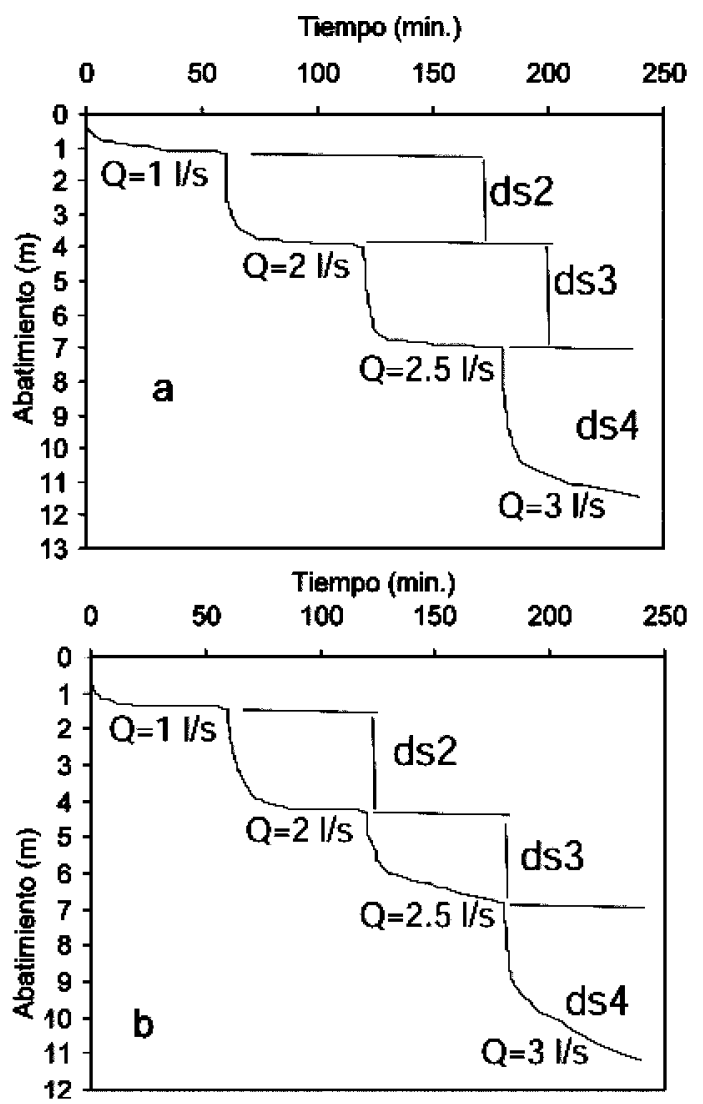

Fig. 4: Abatimiento obtenido antes (a) y después (b) del desarrollo para cada etapa de bombeo en el pozo Tiquirusas de Cañas.

bombeo. Las pérdidas debidas a la resistencia hidráulica estimadas por el método de Jacob son en ambas pruebas mayores que las calculadas por Rorabaugh. No se muestran los resultados por el método analítico, puesto que el análisis numérico de Newton no converge y por lo tanto no se puede determinar la solución a la ecuación. El aumento de la eficiencia después del desarrollo permite un bombeo de $172 \mathrm{~m}^{3} / \mathrm{d}$ para obtener un abatimiento de solo 1,5 m (Fig. 6).

\section{Pozo Cebadillas de Santa Cruz}

Este pozo se ubica en las coordenadas 340,7/24,6 de la hoja cartográfica Villareal del IGN. El material atravesado por la perfo-

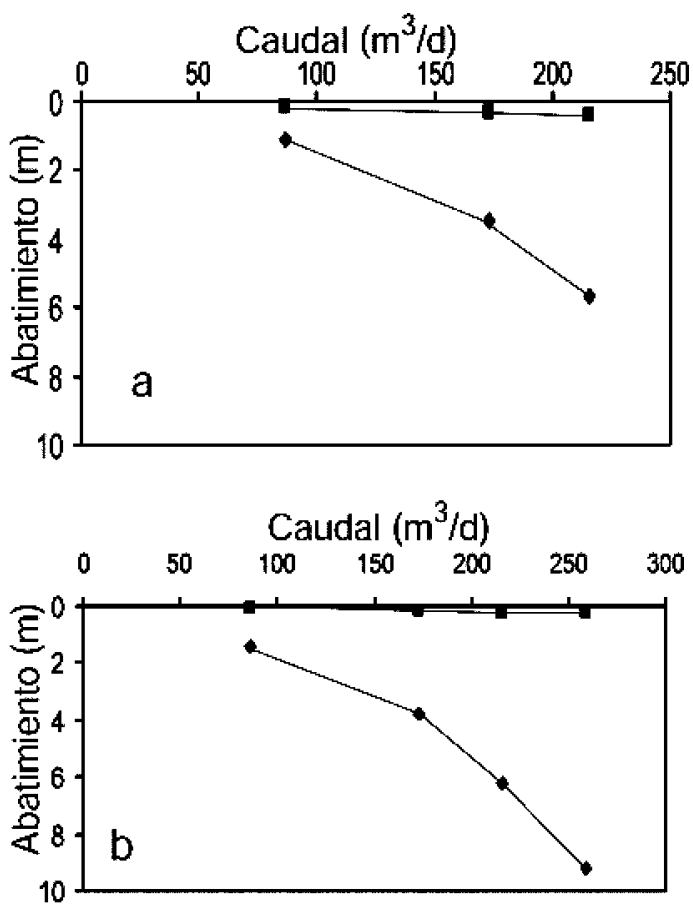

Fig. 5: Curvas de rendimiento en el pozo Tiquirusas de Cañas. Los cuadrados indican los valores teóricos mientras que los rombos representan los valores reales obtenidos en las pruebas de bombeo. a) Antes del desarrollo, b) después del desarrollo.

ración es de tipo volcánico del Complejo de Nicoya. Entre los 0 y 4 metros de profundidad se trata de material arcilloso con características plásticas de muy baja permeabilidad, producto de la meteorización de la roca. Entre los 9 y 28 metros de profundidad la roca presenta algún grado de fracturamiento. La rejilla del pozo se ubica entre los 14 a 27 metros y se refiere a tubería de PVC de ranura continua slot 60 .

La prueba de bombeo por etapas, ejecutada previa al desarrollo, consta de cuatro escalones con un incremento variable en cada uno de ellos. Sin embargo, solo se alcanzó el estado de equilibrio en las etapas iniciales, pero no así en las finales, impidiendo una estimación efectiva de la eficiencia del pozo. El desarrollo tuvo un efecto de disminución de las pérdidas por fricción en el pozo y en la formación que se encuentra alrededor del pozo (Cuadro 4). 
Cuadro 3

Parámetros de prueba por etapas en pozo Río Grande de Nicoya

\begin{tabular}{|c|c|c|c|c|c|c|c|c|c|}
\hline $\mathrm{Q}\left(\mathrm{m}^{3} / \mathrm{d}\right)$ & Tiempo (min) & $\mathrm{sw}(\mathrm{m})$ & swtotal (m) & $\mathrm{n}$ & $\mathrm{B}$ & $\mathrm{C}$ & BQ & $\mathrm{CQ}^{\mathrm{n}}$ & Efic $(\%)$ \\
\hline \multicolumn{7}{|c|}{ Antes del desarrollo 20-5-98 } & \multicolumn{3}{|c|}{ Rorabaugh } \\
\hline 86,4 & 60 & 0,43 & 0,43 & 3,26 & 0,0043 & $3,6 \times 10-8$ & 0,37 & 0,08 & 86,6 \\
\hline 112,32 & 60 & 0,27 & 0,70 & 3,26 & 0,0043 & $3,6 \times 10-8$ & 0,48 & 0,18 & 69,1 \\
\hline 129,6 & 60 & 0,11 & 0,81 & 3,26 & 0,0043 & $3,6 \times 10-8$ & 0,56 & 0,28 & 68,9 \\
\hline 172,8 & 60 & 0,65 & 1,46 & 3,26 & 0,0043 & $3,6 \times 10-8$ & 0,74 & 0,72 & 51,0 \\
\hline \multicolumn{10}{|c|}{ Despúes del desarrollo 21-5-98 } \\
\hline 86,4 & 60 & 0,55 & 0,55 & 2,86 & 0,0056 & $1,8 \times 10-7$ & 0,49 & 0,06 & 88,8 \\
\hline 112,32 & 60 & 0,22 & 0,77 & 2,86 & 0,0056 & $1,8 \times 10-7$ & 0,64 & 0,13 & 82,5 \\
\hline 129,6 & 60 & 0,16 & 0,93 & 2,86 & 0,0056 & $1,8 \times 10-7$ & 0,73 & 0,20 & 78,8 \\
\hline 172,8 & 60 & 0,51 & 1,44 & 2,86 & 0,0056 & $1,8 \times 10-7$ & 0,98 & 0,46 & 68,3 \\
\hline \multicolumn{7}{|c|}{ Antes del desarrollo 20-5-98 } & \multicolumn{3}{|l|}{ Jacob } \\
\hline 86,4 & 60 & 0,43 & 0,43 & 2,0 & 0,0016 & $3,9 \times 10-5$ & 0,14 & 0,29 & 32,0 \\
\hline 112,3 & 60 & 0,27 & 0,70 & 2,0 & 0,0016 & $3,9 \times 10-5$ & 0,18 & 0,49 & 25,6 \\
\hline 129,6 & 60 & 0,11 & 0,81 & 2,0 & 0,0016 & $3,9 \times 10-5$ & 0,21 & 0,65 & 25,5 \\
\hline 172,8 & 60 & 0,65 & 1,46 & 2,0 & 0,0016 & $3,9 \times 10-5$ & 0,28 & 1,15 & 19,1 \\
\hline \multicolumn{10}{|c|}{ Despúes del desarrollo 21-5-98 } \\
\hline 86,4 & 60 & 0,55 & 0,55 & 2,0 & 0,004 & $2,3 \times 10-5$ & 0,36 & 0,18 & 65,9 \\
\hline 172,8 & 60 & 0,21 & 0,76 & 2,0 & 0,004 & $2,3 \times 10-5$ & 0,47 & 0,30 & 62,0 \\
\hline 216,0 & 60 & 0,17 & 0,93 & 2,0 & 0,004 & $2,3 \times 10-5$ & 0,54 & 0,39 & 58,4 \\
\hline 259,2 & 60 & 0,50 & 1,43 & 2,0 & 0,004 & $2,3 \times 10-5$ & 0,72 & 0,70 & 50,7 \\
\hline
\end{tabular}

Q: caudal; sw: abatimiento por etapa; swtotal: abatimiento total acumulado; n: exponente.
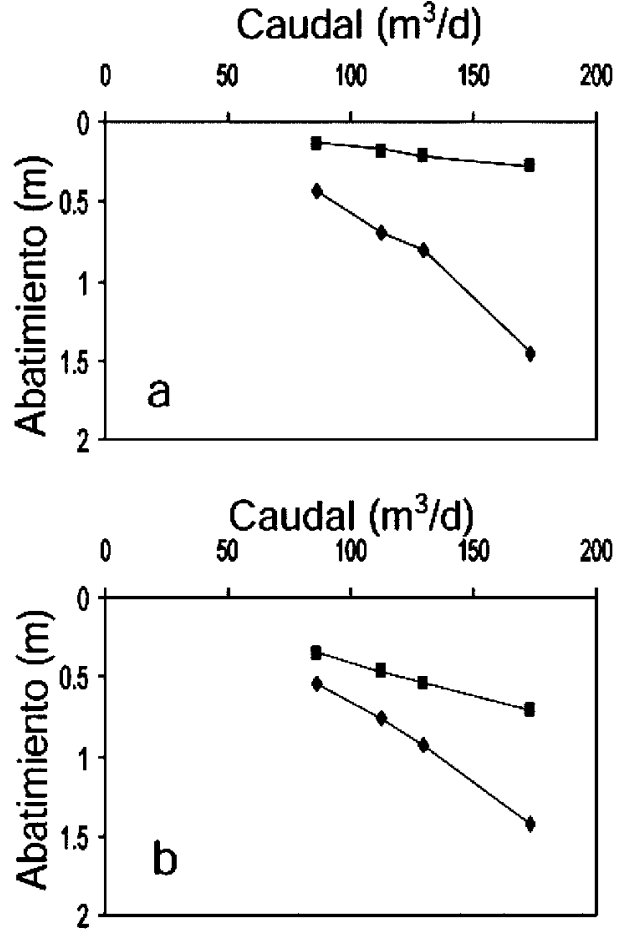

Fig. 6: Curvas de rendimiento en el pozo Río Grande de Nicoya. Los cuadrados indican los valores teóricos mientras que los rombos representan los valores reales obtenidos en las pruebas de bombeo. a) Antes del desarrollo. b) Después del desarrollo.
La eficiencia calculada por el método de Jacob es considerablemente menor que la estimada por Rorabaugh, donde el exponente de Q es 4,85 , lo cual es muy elevado ya que según la literatura este valor varía de 1,5 a 3,5 (Kruseman \& De Ridder, pág. 200). El rendimiento del pozo antes del desarrollo descendió rápidamente con el caudal de $173 \mathrm{~m}^{3} / \mathrm{d}$ hasta casi $4 \mathrm{~m}$ de abatimiento (Fig. 7). Por el método analítico, al igual que el caso anterior, la eficiencia del pozo disminuye después del desarrollo. Además, se nota que los resultados obtenidos son iguales a los obtenidos por el método de Rorabaugh.

\section{Pozo Matapalo de Abangares}

El pozo se ubica en las coordenadas 425,8/247,7 de la hoja Abangares del IGN. El material atravesado es de tipo sedimentario, principalmente arenas finas y medias. El principal estrato productor del acuífero se ubica entre los 15 y $16,5 \mathrm{~m}$ de profundidad y consiste de arenas $\sin$ consolidar de granos entre finos y medios cuya permeabilidad aparente se considera alta. No existe información acerca del armado del pozo. 
Cuadro 4

Parámetros de prueba en pozo Cebadillas de Santa Cruz

\begin{tabular}{|c|c|c|c|c|c|c|c|c|c|}
\hline $\mathrm{Q}\left(\mathrm{m}^{3} / \mathrm{d}\right)$ & Tiempo (min) & $\mathrm{sw}(\mathrm{m})$ & swtotal (m) & $\mathrm{n}$ & B & $\mathrm{C}$ & BQ & $\mathrm{CQ}^{\mathrm{n}}$ & Efic $(\%)$ \\
\hline \multicolumn{7}{|c|}{ Antes del desarrollo 16-6-98 } & \multicolumn{3}{|c|}{ Rorabaugh } \\
\hline 86,4 & 60 & 0,66 & 0,66 & 4,85 & 0,0075 & $4,2 \times 10-12$ & 0,65 & 0,01 & 98,4 \\
\hline 172,8 & 60 & 0,94 & 1,60 & 4,85 & 0,0075 & $4,2 \times 10-12$ & 1,30 & 0,30 & 81,2 \\
\hline 259,2 & 60 & 2,50 & 4,10 & 4,85 & 0,0075 & $4,2 \times 10-12$ & 1,95 & 2,18 & 47,5 \\
\hline 345,6 & 25 & 9,06 & 13,16 & - & - & - & - & - & - \\
\hline \multicolumn{10}{|c|}{ Despúes del desarrollo 23-6-98 } \\
\hline 86,4 & 30 & 0,45 & 0,45 & 3,39 & 0,0044 & $1,87 \times 10-8$ & 0,38 & 0,07 & 85,0 \\
\hline 129,6 & 40 & 0,44 & 0,84 & 3,39 & 0,0044 & $1,87 \times 10-8$ & 0,57 & 0,27 & 68,3 \\
\hline 172,8 & 40 & 0,63 & 1,47 & 3,39 & 0,0044 & $1,87 \times 10-8$ & 0,77 & 0,71 & 52,1 \\
\hline \multicolumn{7}{|c|}{ Antes del desarrollo 16-6-98 } & \multicolumn{3}{|c|}{ Jacob } \\
\hline 86,4 & 60 & 0,66 & 0,66 & 2,00 & 0,00017 & $5,9 \times 10-5$ & 0,01 & 0,44 & 2,2 \\
\hline 172,8 & 60 & 0,94 & 1,60 & 2,00 & 0,00017 & $5,9 \times 10-5$ & 0,03 & 1,77 & 1,8 \\
\hline 259,2 & 60 & 2,50 & 4,10 & 2,00 & 0,00017 & $5,9 \times 10-5$ & 0,04 & 3,99 & 1,1 \\
\hline 345,6 & 25 & 9,06 & 13,16 & - & - & - & - & - & - \\
\hline \multicolumn{10}{|c|}{ Despúes del desarrollo 23-6-98 } \\
\hline 86,4 & 60 & 0,45 & 0,45 & 2,00 & 0,0015 & $4,0 \times 10-5$ & 0,13 & 0,30 & 28,3 \\
\hline 129,6 & 60 & 0,39 & 0,84 & 2,00 & 0,0015 & $4,0 \times 10-5$ & 0,19 & 0,68 & 22,8 \\
\hline 172,8 & 60 & 0,63 & 1,47 & 2,00 & 0,0015 & $4,0 \times 10-5$ & 0,25 & 1,2 & 17,3 \\
\hline \multicolumn{7}{|c|}{ Antes del desarrollo 16-6-98 } & \multicolumn{3}{|c|}{ Mét. analítico } \\
\hline 86.4 & 60 & 0,66 & 0,66 & 4,85 & 0.0075 & $4,2 \times 10-12$ & 0,649 & 0,0104 & 98,4 \\
\hline 172.8 & 60 & 0,94 & 1,60 & 4,85 & 0.0075 & $4,2 \times 10-12$ & 1,299 & 0,30 & 81,2 \\
\hline 259.2 & 60 & 2,50 & 4,10 & 4,85 & 0.0075 & $4,2 \times 10-12$ & 1,948 & 2,151 & 47,5 \\
\hline \multicolumn{10}{|c|}{ Despúes del desarrollo 23-6-98 } \\
\hline 86,4 & 30 & 0,45 & 0,45 & 3,39 & 0,0044 & $1,90 \times 10-8$ & 0,382 & 0,0675 & 85,0 \\
\hline 129,6 & 40 & 0,44 & 0,84 & 3,39 & 0,0044 & $1,90 \times 10-8$ & 0,574 & 0,266 & 68,3 \\
\hline 172,8 & 40 & 0,63 & 1,47 & 3,39 & 0,0044 & $1,87 \times 10-8$ & 0,7665 & 0,705 & 52,1 \\
\hline
\end{tabular}

Q: caudal; sw: abatimiento por etapa; swtotal: abatimiento total acumulado; n: exponente.

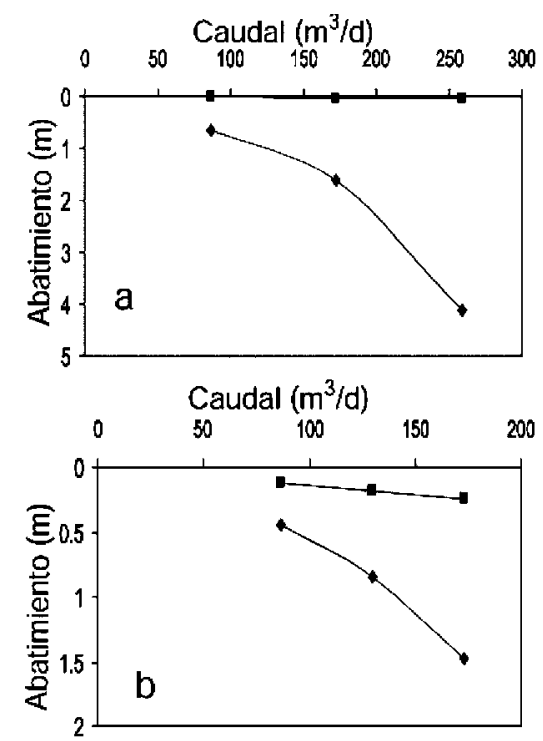

Fig. 7: Curvas de rendimiento en el pozo Cebadillas de Santa Cruz. Los cuadrados indican los valores teóricos mientras que los rombos representan los valores reales obtenidos en las pruebas de bombeo.a) Antes del desarrollo. b) Después del desarrollo.
Se tienen datos de dos pruebas de bombeo cada una de cuatro etapas y con una duración de 60 minutos (Cuadro 5). En las etapas 1, 2 y 3 de la prueba previa al desarrollo se alcanzó una condición de estado estacionario, mientras que en la prueba posterior solo se alcanzó el equilibrio en las etapas 1 y 2. La eficiencia calculada por el método de Rorabaugh en la prueba previa al desarrollo es superior a la calculada por Jacob, sin embargo, en la prueba posterior la eficiencia por Jacob superó a Rorabaugh. Las curvas de rendimiento del pozo muestran que el abatimiento máximo esperado para un caudal de $173 \mathrm{~m}^{3} / \mathrm{d}$ es de $2 \mathrm{~m}$ y se pronostica un abatimiento ideal de solo $1 \mathrm{~m}$ (Fig. 8).

\section{Pozo Piedras Verdes de Abangares}

El pozo se ubica en las coordenadas 422,6/149,3 de la hoja topográfica Abangares editada por el IGN. A dos kilómetros del sitio se 
Cuadro 5

Parámetros de prueba en pozo Matapalo de Abangares

\begin{tabular}{|c|c|c|c|c|c|c|c|c|c|}
\hline $\mathrm{Q}\left(\mathrm{m}^{3} / \mathrm{d}\right)$ & Tiempo (min) & sw (m) & swtotal (m) & $\mathrm{n}$ & $\mathrm{B}$ & $\mathrm{C}$ & BQ & $\mathrm{CQ}^{2}$ & Efic $(\%)$ \\
\hline \multicolumn{7}{|c|}{ Antes del desarrollo 11-8-98 } & \multicolumn{3}{|c|}{ Rorabaugh } \\
\hline 43,2 & 60 & 0,14 & 0,14 & 2,46 & 0,0031 & $4,7 \times 10-6$ & 0,13 & 0,05 & 94,9 \\
\hline 86,4 & 60 & 0,46 & 0,60 & 2,46 & 0,0031 & $4,7 \times 10-6$ & 0,27 & 0,27 & 44,3 \\
\hline 129,6 & 60 & 0,49 & 1,09 & 2,46 & 0,0031 & $4,7 \times 10-6$ & 0,40 & 0,74 & 36,6 \\
\hline 172,8 & 60 & 0,94 & 2,03 & 2,46 & 0,0031 & $4,7 \times 10-6$ & 0,53 & 1,5 & 26,2 \\
\hline \multicolumn{10}{|c|}{ Despúes del desarrollo 11-8-98 } \\
\hline 43,2 & 60 & 0,25 & 0,25 & 1,87 & $5,4 \times 10-3$ & $8,6 \times 10-5$ & 0,23 & 0,1 & 93,7 \\
\hline 86,4 & 60 & 0,70 & 0,97 & 1,87 & $5,4 \times 10-3$ & $8,6 \times 10-5$ & 0,47 & 0,35 & 48,3 \\
\hline 129,6 & 60 & 0,38 & 1,33 & 1,87 & $5,4 \times 10-3$ & $8,6 \times 10-5$ & 0,70 & 0,76 & 52,8 \\
\hline 172,8 & 60 & 0,92 & 2,25 & 1,87 & $5,4 \times 10-3$ & $8,6 \times 10-5$ & 0,94 & 1,29 & 41,6 \\
\hline \multicolumn{9}{|c|}{ Antes del desarrollo 11-8-98 } & Jacob \\
\hline 43,2 & 60 & 0,14 & 0,14 & 2,0 & $1,8 \times 10-3$ & $5,6 \times 10-5$ & 0,08 & 0,10 & 62,1 \\
\hline 86,4 & 60 & 0,46 & 0,60 & 2,0 & $1,8 \times 10-3$ & $5,6 \times 10-5$ & 0,16 & 0,42 & 23,1 \\
\hline 129,6 & 60 & 0,49 & 1,09 & 2,0 & $1,8 \times 10-3$ & $5,6 \times 10-5$ & 0,24 & 0,94 & 22,4 \\
\hline 172,8 & 60 & 0,94 & 2,03 & 2,0 & $1,8 \times 10-3$ & $5,6 \times 10-5$ & 0,32 & 1,67 & 15,9 \\
\hline \multicolumn{10}{|c|}{ Despúes del desarrollo 11-8-98 } \\
\hline 43,2 & 60 & 0,25 & 0,25 & 2,0 & $6,1 \times 10-3$ & $3,9 \times 10-5$ & 0,26 & 0,07 & 100,0 \\
\hline 86,4 & 60 & 0,70 & 0,97 & 2,0 & $6,1 \times 10-3$ & $3,9 \times 10-5$ & 0,53 & 0,29 & 54,4 \\
\hline 129,6 & 60 & 0,38 & 1,33 & 2,0 & $6,1 \times 10-3$ & $3,9 \times 10-5$ & 0,79 & 0,65 & 59,6 \\
\hline 172,8 & 60 & 0,92 & 2,25 & 2,0 & $6,1 \times 10-3$ & $3,9 \times 10-5$ & 1,06 & 1,16 & 46,9 \\
\hline
\end{tabular}

Q: caudal; sw: abatimiento por etapa; swtotal: abatimiento total acumulado; n: exponente.

cuenta con información de los pozos $\mathrm{AN}-85$ y AN-86. De 0-6 m se presenta una arcilla roja de comportamiento plástico, de $6-14 \mathrm{~m}$ limo y arena fina, de 14-24 m un horizonte de gravas y arenas envueltos en una matriz arcillosa y finalmente, de 24-50 m se tiene una lava andesítica masiva, silicificada de bajo grado de fracturación. El principal estrato productor de agua subterránea se ubica entre 14-24 $\mathrm{m}$ de profundidad y se refiere al horizonte de gravas y arenas con una permeabilidad aparente alta.

En este pozo se dispone de 2 pruebas de bombeo escalonadas. La primera prueba consta de dos etapas de 60 minutos cada una y la tercera es de solo 3,5 minutos, lo cual es insuficiente para estimar adecuadamente la eficiencia del pozo por el método de Rorabaugh, por lo que se ha calculado la eficiencia con el método de Jacob, obteniéndose valores que varían de $74 \%$ a $63 \%$ antes del desarrollo. La eficiencia calculada con

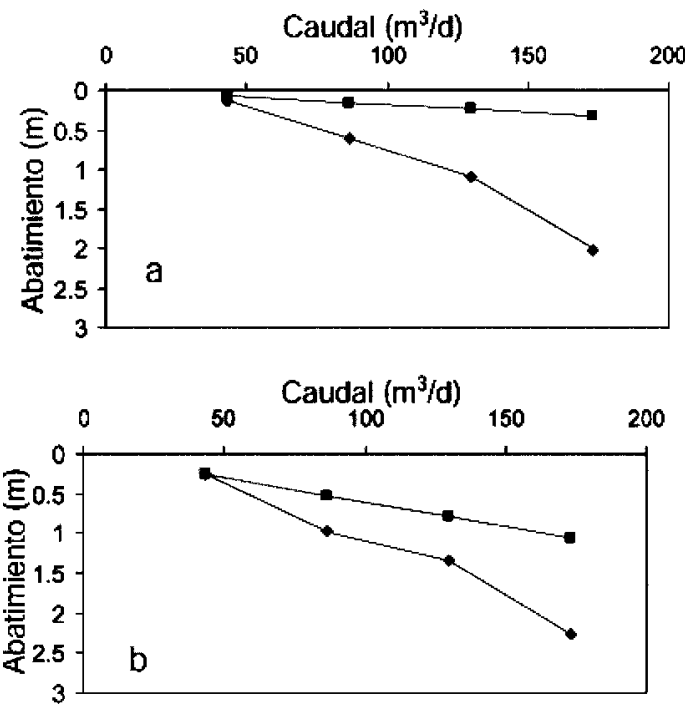

Fig. 8: Curvas de rendimiento en el pozo Matapalo de Abangares. Los cuadrados indican los valores teóricos mientras que los rombos representan los valores reales obtenidos en las pruebas de bombeo. a) Antes del desarrollo. b) Después del desarrollo. 
los datos de la prueba posterior al desarrollo es similar por los dos métodos, variando de 43 a 60 $\%$, notándose que ha ocurrido un descenso en la eficiencia (Cuadro 6), debido posiblemente a que en el ultimo escalón de la etapa previa al desarrollo el lapso de bombeo fue muy corto y afectó la estimación. Walton (1991, pág. 164) recomienda que los cambios en el abatimiento se determinan tomando en cuenta períodos de bombeo de igual duración. Por medio del método analítico los resultados obtenidos son iguales a los del método de Rorabaugh, notándose otra vez una disminución de la eficiencia, más pronunciada conforme aumenta el caudal de extracción. Después del desarrollo es posible obtener un abatimiento de $4 \mathrm{~m}$ con un caudal de $172 \mathrm{~m}^{3} / \mathrm{d}$ (Fig. 9).

\section{DISCUSIÓN DE RESULTADOS}

Los métodos pueden mostrar diferencias entre sí en los porcentajes de eficiencia calculados para las etapas previas y posteriores al desarrollo. Así, por ejemplo, con los métodos de Rorabaugh y analítico se observa para las pruebas realizadas en el pozo Tiquirusas un incremento leve de la eficiencia; no obstante, con el método
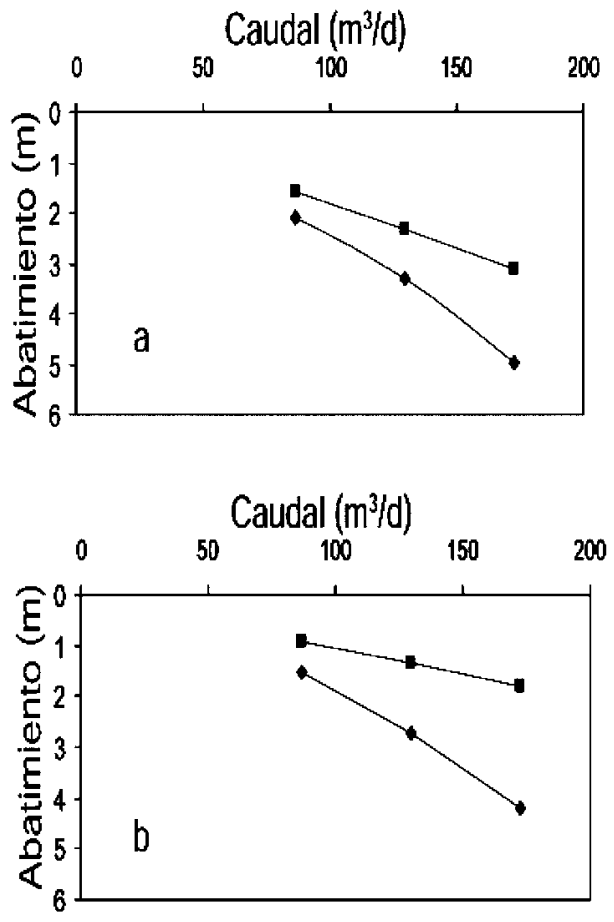

Fig. 9: Curvas de rendimiento en el pozo Piedras Verdes de Abangares. Los cuadrados indican los valores teóricos mientras que los rombos representan los valores reales obtenidos en las pruebas de bombeo. a) Antes del desarrollo. b) después del desarrollo.

Cuadro 6

Parámetros de prueba en pozo Piedras Verdes de Abangares

\begin{tabular}{|c|c|c|c|c|c|c|c|c|c|}
\hline $\mathrm{Q}\left(\mathrm{m}^{3} / \mathrm{d}\right)$ & Tiempo (min) & sw (m) & Swtotal (m) & $\mathrm{n}$ & $\mathrm{B}$ & $\mathrm{C}$ & BQ & $\mathrm{CQ}^{\mathrm{n}}$ & Efic. (\%) \\
\hline \multicolumn{7}{|c|}{ Después del desarrollo 11-8-98 } & \multicolumn{3}{|c|}{ Rorabaugh } \\
\hline 86,4 & 60 & 1,5 & 1,50 & 2,0 & 0,01 & $8,0 \times 10-5$ & 0,90 & 0,61 & 60,0 \\
\hline 129,6 & 60 & 1,2 & 2,70 & 2,0 & 0,01 & $8,0 \times 10-5$ & 1,35 & 1,36 & 50,0 \\
\hline 172,8 & 60 & 1,5 & 4,20 & 2,0 & 0,01 & $8,0 \times 10-5$ & 1,80 & 2,42 & 42,9 \\
\hline \multicolumn{7}{|c|}{ Antes del desarrollo 11-8-98 } & \multicolumn{3}{|c|}{ Jacob } \\
\hline 86,4 & 60 & 2,1 & 2,08 & 2,0 & 0,018 & $6,1 \times 10-5$ & 1,56 & 0,46 & 74,8 \\
\hline 129,6 & 60 & 1,2 & 3,28 & 2,0 & 0,018 & $6,1 \times 10-5$ & 2,33 & 1,03 & 71,2 \\
\hline 172,8 & 3,5 & 1,7 & 4,98 & 2,0 & 0,018 & $6,1 \times 10-5$ & 3,11 & 1,83 & 62,5 \\
\hline \multicolumn{10}{|c|}{ Despúes del desarrollo 11-8-98 } \\
\hline 86,4 & 60 & 1,5 & 1,50 & 2,0 & 0,01 & $8,0 \times 10-5$ & 0,90 & 0,60 & 60,0 \\
\hline 129,6 & 60 & 1,2 & 2,70 & 2,0 & 0,01 & $8,0 \times 10-5$ & 1,35 & 1,35 & 50,0 \\
\hline 172,8 & 60 & 1,5 & 4,20 & 2,0 & 0,01 & $8,0 \times 10-5$ & 1,80 & 2,40 & 42,9 \\
\hline \multicolumn{7}{|c|}{ Después del desarrollo 11-8-98 } & \multicolumn{3}{|c|}{ Mét. analítico } \\
\hline 86,4 & 60 & 1,5 & 1,50 & 2,0 & 0,01 & $8,0 \times 10-5$ & 0,90 & 0,61 & 60,0 \\
\hline 129,6 & 60 & 1,2 & 2,70 & 2,0 & 0,01 & $8,0 \times 10-5$ & 1,35 & 1,36 & 50,0 \\
\hline 172,8 & 60 & 1,5 & 4,20 & 2,0 & 0,01 & $8,0 \times 10-5$ & 1,80 & 2,42 & 42,9 \\
\hline
\end{tabular}

Q: caudal; sw: abatimiento por etapa; swtotal: abatimiento total acumulado; n: exponente. 
de Jacob hay un descenso de la eficiencia. De manera similar, para las etapas previas y posteriores al desarrollo en el pozo Cebadillas de Santa Cruz existe un descenso en la eficiencia mostrada por los métodos de Rorabaugh y analítico. Sin embargo, por el método de Jacob ocurre un incremento de la eficiencia. En las etapas de bombeo realizadas en los pozos de río Grande de Nicoya y Matapalo de Abangares ocurre una coincidencia en cuanto al incremento de la eficiencia estimada por el método de Rorabaugh y Jacob. Se nota que en algunos casos los resultados son diferentes entre los métodos, como por ejemplo en los pozos de Tiquirusas y Cebadillas. Surge entonces la interrogante en cual método es recomendable confiar.

Karanjac (1972, en Braticevic \& Karanjac, 1994) demostraron que la pérdida de carga en un pozo no es necesariamente proporcional a la segunda potencia del caudal de bombeo. Las pérdidas en el pozo podrían ser significantes aunque todavía no se presente el proceso de turbulencia. Por lo tanto, los métodos de Rorabaugh y analítico representan una mejor aproximación. Sin embargo, el método de Jacob permite calcular la eficiencia de una manera más expedita, sin la necesidad de cumplir el requerimiento de los otros métodos en cuanto al incremento en las diferencias de abatimiento entre etapas de bombeo.

Para el pozo de Piedras Verdes de Abangares el método de Jacob indica una disminución de la eficiencia en casi un $20 \%$ entre las etapas previas y posteriores al desarrollo. Con los métodos de Rorabaugh y analítico no es posible calcular la eficiencia ya que no hay incrementos en las diferencias de abatimiento en las etapas previas al desarrollo, lo cual es una desventaja de estos métodos. En el caso de Rorabaugh y analítico el algoritmo de ajuste se desempeña eficientemente cuando los abatimientos van sucediendo en orden creciente con la diferencia entre abatimientos, siendo progresivamente más grandes para incrementos del caudal de bombeo. Se espera además, que la capacidad específica del pozo disminuya con un incremento del caudal de bombeo. Para el caso del pozo de Piedras Verdes esto no ocurre así, por lo que el algoritmo de solución presenta un error de aplicación.
Por otro lado, se ha encontrado que valores de $\mathrm{n}$ menores o cercanos a 2 se presentan cuando se realizan pruebas con caudales bajos, como en el pozo Matapalo de Abangares, donde es probable que la turbulencia a la entrada de la rejilla no se desarrollara completamente. Por el contrario, cuando en alguna de las etapas se bombean caudales relativamente grandes $\left(345 \mathrm{~m}^{3} / \mathrm{d}\right)$, como en el caso del pozo Cebadillas de Santa Cruz, se obtienen valores de $\mathrm{n}$ grandes $(4,85)$, por lo cual el pozo pierde eficiencia, lo que demuestra la relación directa entre el aumento de caudal y la pérdida de eficiencia, lo que permite verificar lo que ya se conocía en la literatura técnica. Por otro lado, se encontró que los métodos de Rorabaugh y analítico son más sensibles a las pérdidas reales de eficiencia generadas por caudales elevados, lo que representa una ventaja de dichos métodos sobre el de Jacob.

La observación de los resultados indica que el valor de $\mathrm{C}$ calculado por el método de Jacob es mayor que los valores estimados por los otros métodos. Así mismo, el valor de B es más bajo, tanto para las etapas previas y posteriores al desarrollo. Esto sucede porque se asume que $n$ es igual a dos, lo cual se traduce en una mayor inclinación de la recta de mejor ajuste a partir de donde se obtienen los valores de B y C.

\section{CONCLUSIONES Y RECOMENDACIONES}

El método de Jacob permite estimar la eficiencia de una manera más expedita pero es recomendable tener las condiciones adecuadas durante la ejecución del ensayo de bombeo escalonado. Se ha observado que una pequeña variación en la estimación del abatimiento de cada etapa provoca cambios drásticos en el valor de la eficiencia calculada.

Los resultados obtenidos sugieren que el desarrollo por cualquiera de los dos procedimientos empleados, es decir, aire comprimido o hielo seco, provoca un aumento en la eficiencia del pozo. Sin embargo, no es posible compararlos en cuanto al grado de aumento de la eficiencia, pues ésta depende de varios factores, entre 
ellos el armado del pozo. Para los casos analizados aquí, no se dispone siempre de la información respectiva, de tal manera que se puedan realizar valoraciones usando pozos de diseño similar. No obstante, los resultados sugieren que el método de aire comprimido provoca un aumento mayor de la eficiencia del pozo.

En el pozo Cebadillas de Santa Cruz se obtuvo un descenso en la eficiencia después del desarrollo indicado por los métodos de Rorabaugh y analítico, que se debe al elevado caudal de extracción empleado durante algunas etapas de bombeo o al reacomodo de los materiales finos alrededor de la rejilla colmatando los poros de la formación acuífera o a una deformación de la rejilla que limita la entrada de agua al pozo.

Para el cálculo de la eficiencia por los métodos de Rorabaugh y analítico se requiere al menos la ejecución de una prueba con tres etapas de bombeo con un incremento en la diferencia entre abatimientos. El valor de n, según estos métodos para las pruebas analizadas, varía de 1,87 a 4,85 .

El abatimiento de cada etapa de bombeo se debe considerar como la diferencia entre el valor inicial del nivel estático y el nivel estático al final de la etapa, si esto no sucediera se puede proyectar una línea de abatimiento probable. Este procedimiento se simplifica con un gráfico de abatimiento versus tiempo, donde se puede dibujar la proyección del abatimiento.

La estimación de la eficiencia por medio de los métodos utilizados aquí es válida para acuíferos libres cuando el descenso del nivel de agua subterránea es despreciable comparado con el espesor saturado inicial.
El método analítico desarrollado en este trabajo tiene entre sus ventajas, que se puede implementar en una calculadora de bolsillo programable y permite obtener la eficiencia de manera más expedita y confiable.

Como recomendación se sugiere que durante los ensayos de bombeo escalonados se realicen las mediciones del abatimiento con precisión y esperar a que se alcance el estado estacionario del nivel de agua subterránea de cada etapa para proceder a calcular la eficiencia de una manera más acertada. De no ser así se pueden obtener valores de B negativos o los valores de eficiencia no serían muy confiables.

\section{REFERENCIAS}

BRATICEVIC, D. \& KARANJAC, J., 1994: Groundwater software for windows. Users Manual. - 405 págs. Naciones Unidas, Nueva York.

CUSTODIO, M. \& LLAMAS, R., 1983: Hidrología subterránea. - 1800 págs. Tomo I. Omega, Madrid.

DEPARTAMENTO AGUAS SUBTERRÁNEAS, AyA., 2001: Archivo de pozos. - Instituto Costarricense de Acueductos y Alcantarillados. San José.

KRUSEMAN, G.P. \& De RIDDER, N.A., 1994: Analysis and evaluation of pumping test data. - 377 págs. Internat. Inst. Land Reclamation, Wageningen.

LANGGUTH, H.R. \& VOIGT, R., 1980: Hydrogeologische Methoden. - 486 págs. Springer, Berlin.

WALTON, W., 1970: Groundwater resource evaluation. - 663 págs. Mc Graw-Hill, Nueva York.

WALTON, W., 1991: Principles of groundwater engineering. - 546 págs. Lewis Publ. Michigan. 